\title{
CONNECTION COEFFICIENTS OF SHANNON WAVELETS ${ }^{1}$
}

\author{
C. CATTANI \\ diFARMA, University of Salerno
}

Via Ponte Don Melillo, 84084 Fisciano (SA) Italy

E-mail: ccattani@unisa.it

Received February 15, 2006; revised April 15, 2006; published online May 25, 2006

\begin{abstract}
In this paper the differential properties of Shannon wavelets are investigated. The connection coefficients of Shannon wavelets are explicitly computed with a finite formula up to any order.
\end{abstract}

Key words: Harmonic Wavelets, Shannon Wavelets, connection coefficients

\section{Introduction}

In some previous papers $[2,3,4,11,13]$, it has been shown that in order to study the evolution of solitary wave profiles it is expedient to make use of wavelets with finite support and analytically defined. Complex harmonic wavelets [14] were applied to the solution of evolution problems $[2,3,4,13]$. In particular, in [13] the solution of the Burgers equation was given by using the connection coefficients for periodised harmonic wavelets. This method shortly consists in the Galerkin-Petrov numerical solution of a partial differential system (see e.g. $[7,8]$ ), so that the connection coefficients are the projection of the various order derivatives of the basis functions along with the orthonormal wavelet basis. By using the Galerkin-Petrov method in an orthonormal basis the connection coefficients (also called refinable integrals) follow from the scalar product of the basis functions with their derivatives (see e.g. [10]).

Harmonic wavelets are complex functions and band-limited in the Fourier domain $[1,5,11,12,14]$, so that they can be used to study frequency changes as well as oscillations in a small range time interval. However, in practical real

\footnotetext{
${ }^{1}$ This work has been partially supported by Regione Campania under contracts: "Modelli nonlineari di materiali compositi per applicazioni di nanotecnologia chimica-biomedica". LR 28/5/02 n. 5, Finanziamenti 2003, and by D.G.R. n. $2534,30 / 12 / 04$.
} 
problems, it seems to be more natural to make use of real wavelet functions. In particular, Shannon wavelets, which are derived from the real part of the harmonic wavelets, are a family of real functions. Their connection coefficients will be explicitly computed and it will be shown that they fulfill some recursive formulas, thus making an easy computation up to any order.

In general the computation of the connection coefficients seems to be a difficult task for two reasons: first, the most known (and used) wavelets are not functionally defined by a finite formula and, second, even in presence of a simple formula defining the wavelet family, such as the Daubechies family [9], there does not exist a simple expression for the corresponding connection coefficients [11]. Moreover, except for the first and second derivative, there were not explicit formulas for higher derivatives (for the connection coefficients of harmonic wavelets see $[2,3,6])$.

Although the investigation of non-orthonormal wavelet bases (and corresponding connection coefficients) would be a more interesting problem as well as the numerical investigation of PDE solution by wavelet method [6] (with special treatment of boundary conditions), in the following we will focus only on the connection coefficients of the Shannon wavelet, by giving for the first time a finite formula for their computation. It should be noticed that the Shannon connection coefficients can be easily defined but their explicit values usually require lengthy computations. Only by using a computer algebra symbolic system it was possible to obtain a finite formula for calculating their numerical values. In fact, this formula is not evident a priori but it can be summarized only after the computation of a large amount of numerical sequence.

\section{Harmonic Wavelets}

The harmonic scaling function $[5,6,14]$

$$
\varphi(x) \stackrel{\text { def }}{=} \frac{e^{2 \pi i x}-1}{2 \pi i x}
$$

is a complex function. The real and imaginary parts of this function are given by

$$
\Re(\varphi(x))=\frac{\sin 2 \pi x}{2 \pi x}, \quad \Im(\varphi(x))=\frac{\sin ^{2} \pi x}{\pi x} .
$$

Since

$$
e^{\pi i n}=\left\{\begin{array}{l}
1, \quad n=2 k, \quad k \in \mathbb{Z}, \\
-1, \quad n=2 k+1, \quad k \in \mathbb{Z},
\end{array}\right.
$$

it is, in particular,

$$
\varphi(n)=0, \quad n \in \mathbb{Z} .
$$


The harmonic scaling function (2.1) was defined [14] in such a way that its Fourier transform

$$
\widehat{\varphi}(\omega)=\widehat{\varphi(x)} \stackrel{\text { def }}{=} \frac{1}{2 \pi} \int_{-\infty}^{\infty} \varphi(x) e^{-i \omega x} \mathrm{~d} x
$$

results with compact support in the frequency domain (i.e. with bounded frequency)

$$
\widehat{\varphi}(\omega)=\frac{1}{2 \pi} \chi(2 \pi+\omega) .
$$

The characteristic (or box) function $\chi(\omega)$ is defined in the following way

$$
\chi(\omega) \stackrel{\text { def }}{=} \begin{cases}1, & 2 \pi \leq \omega \leq 4 \pi, \\ 0, & \text { elsewhere. }\end{cases}
$$

It is a very well localized function in the frequency domain, despite its slow decay in the space variable.

Starting from the scaling function it is possible to get a filter and to derive the corresponding multiresolution analysis (see e.g. [14]). The harmonic wavelet is the complex valued function $[13,14]$

$$
\psi(x) \stackrel{\text { def }}{=} \frac{e^{4 \pi i x}-e^{2 \pi i x}}{2 \pi i x}=e^{2 \pi i x} \varphi(x),
$$

and, the dilated and translated instances [2, 4], are

$$
\psi_{k}^{n}(x) \stackrel{\text { def }}{=} 2^{n / 2} \frac{e^{4 \pi i\left(2^{n} x-k\right)}-e^{2 \pi i\left(2^{n} x-k\right)}}{2 \pi i\left(2^{n} x-k\right)},
$$

with $n, k \in \mathbb{Z}$. Analogously, for each function of the wavelet family (2.5), it is

$$
\left|\psi_{k}^{n}(x)\right|=\left|\frac{\sin \pi\left(2^{n} x-k\right)}{\pi\left(2^{n} x-k\right)}\right|,
$$

so that $\lim _{n, k, x \rightarrow \infty}\left|\psi_{k}^{n}(x)\right|=0$. The Fourier transform of (2.5), (see e.g. [14]) are the band-limited functions

$$
\widehat{\psi}_{k}^{n}(\omega)=\frac{2^{-n / 2}}{2 \pi} e^{-i \omega k / 2^{n}} \chi\left(\omega / 2^{n}\right),
$$

being, in particular, $\widehat{\psi}(\omega)=\frac{1}{2 \pi} \chi(\omega)$. In the derivation of equation (2.6) we have taken into account some properties of the Fourier transform, i.e. if $\widehat{f}(\omega)$ is the Fourier transform of $f(x)$ then

$$
f(\widehat{a x \pm} b)=\frac{1}{a} e^{ \pm i \omega b / a} \widehat{f}\left(\frac{\omega}{a}\right) .
$$


From the definition of the scalar product, of two functions $f(x), g(x)$, and taking into account the Parseval equality, it is

$$
\langle f, g\rangle \stackrel{\text { def }}{=} \int_{-\infty}^{\infty} f(x) \overline{g(x)} \mathrm{d} x=2 \pi \int_{-\infty}^{\infty} \widehat{f}(\omega) \overline{\widehat{g}(\omega)} \mathrm{d} \omega=2 \pi\langle\widehat{f}, \widehat{g}\rangle,
$$

where the bar stands for the complex conjugate. It can be shown that harmonic wavelets are orthonormal functions $[2,6,14]$, in the sense that $\left\langle\psi_{k}^{n}(x), \psi_{h}^{m}(x)\right\rangle=\delta^{n m} \delta_{h k}$, and $\left\langle\psi_{k}^{n}(x), \overline{\psi_{h}^{m}}(x)\right\rangle=\delta^{n m} \delta_{h k}, \delta^{n m}, \delta_{h k}$ being the Kronecker symbols.

\subsection{Preliminary formulas}

In order to define any order differential properties of Shannon wavelets, in the following we will meet the integrals $\int_{2 \pi}^{4 \pi} \xi^{\ell} e^{i m \xi} \mathrm{d} \xi$. Usually they might be expressed as

$$
\int_{2 \pi}^{4 \pi} \xi^{\ell} e^{i m \xi} \mathrm{d} \xi=\frac{1}{(-i m)^{\ell+1}}[\Gamma(\ell+1, \xi)]_{-4 i m \pi}^{-2 i m \pi}
$$

being $\Gamma(\ell, \xi) \stackrel{\text { def }}{=} \int_{\xi}^{\infty} t^{\ell-1} e^{-t} \mathrm{~d} t$ the Euler Gamma function, and $[F(\xi)]_{\xi_{0}}^{\xi_{1}} \stackrel{\text { def }}{=}$ $F\left(\xi_{1}\right)-F\left(\xi_{0}\right)$. However, it is possible to give them an explicit recursive formula as follows.

Theorem 1. For a given $m \in \mathbb{Z}, \ell \in \mathbb{N} \cup\{0\}$, and $\xi \in[a, b] \subset \mathbb{R}$, it is

$$
\int_{a}^{b} \xi^{\ell} e^{i m \xi} d \xi=\left\{\begin{array}{l}
-\frac{\ell}{i m} \int_{a}^{b} \xi^{\ell-1} e^{i m \xi} d \xi+\left[\frac{1}{i m} \xi^{\ell} e^{i m \xi}\right]_{a}^{b}, \quad \ell \geq 1 \\
{\left[\frac{1}{i m} e^{i m \xi}\right]_{a}^{b}, \quad \ell=0 .}
\end{array}\right.
$$

Proof. It can be easily obtained by partial integration.

In particular,

Theorem 2. For a given $m \in \mathbb{Z} /\{0\}$ and $\ell \in \mathbb{N} \cup\{0\}$, it is

$$
\int_{2 \pi}^{4 \pi} \xi^{\ell} e^{i m \xi} d \xi=\sum_{k=1}^{\ell}(-1)^{\ell-k} \frac{\ell !(2 \pi)^{k}\left(2^{k}-1\right)}{k !(i m)^{\ell-k+1}}, \quad \ell \geq 1
$$

and

$$
\int_{2 \pi}^{4 \pi} \xi^{\ell} e^{i m \xi} d \xi=0, \quad \ell=0
$$


Proof. According to theorem 1, it is

$$
\left\{\begin{array}{l}
\int_{2 \pi}^{4 \pi} \xi^{\ell} e^{i m \xi} \mathrm{d} \xi=-\frac{\ell}{i m} \int_{2 \pi}^{4 \pi} \xi^{\ell-1} e^{i m \xi} \mathrm{d} \xi+\left[\frac{1}{i m} \xi^{\ell} e^{i m \xi}\right]_{2 \pi}^{4 \pi}, \quad \ell \geq 1 \\
\int_{2 \pi}^{4 \pi} \xi^{\ell} e^{i m \xi} \mathrm{d} \xi=0, \quad \ell=0 .
\end{array}\right.
$$

By defining $I^{\ell} \stackrel{\text { def }}{=} \int_{2 \pi}^{4 \pi} \xi^{\ell} e^{i m \xi} \mathrm{d} \xi$, and taking into account (2.3), we have the recursive formula

$$
I^{0}=0 \quad, \quad I^{\ell}=\frac{-\ell}{i m} I^{\ell-1}+\left[\frac{1}{i m} \xi^{\ell} e^{i m \xi}\right]_{2 \pi}^{4 \pi}, \quad \ell \geq 1 .
$$

We can write explicit results

$$
\left\{\begin{aligned}
I^{0}= & 0, \quad I^{1}=\left[\frac{1}{i m} \xi\right]_{2 \pi}^{4 \pi}, \\
I^{2}= & -\frac{2}{i m}\left[\frac{1}{i m} \xi\right]_{2 \pi}^{4 \pi}+\left[\frac{1}{i m} \xi^{2}\right]_{2 \pi}^{4 \pi}, \\
I^{3}= & -\frac{3}{i m}\left\{-\frac{2}{i m}\left[\frac{1}{i m} \xi\right]_{2 \pi}^{4 \pi}+\left[\frac{1}{i m} \xi^{2}\right]_{2 \pi}^{4 \pi}\right\}+\left[\frac{1}{i m} \xi^{3}\right]_{2 \pi}^{4 \pi}, \\
I^{4}= & -\frac{4}{i m}\left\{-\frac{3}{i m}\left\{-\frac{2}{i m}\left[\frac{1}{i m} \xi\right]_{2 \pi}^{4 \pi}+\left[\frac{1}{i m} \xi^{2}\right]_{2 \pi}^{4 \pi}\right\}+\left[\frac{1}{i m} \xi^{3}\right]_{2 \pi}^{4 \pi}\right\} \\
& +\left[\frac{1}{i m} \xi^{4}\right]_{2 \pi}^{4 \pi}, \\
& \ldots . . . . . . . . . . .
\end{aligned}\right.
$$

So that we can write

$$
\left\{\begin{aligned}
I^{0}= & 0, \quad I^{1}=\left[\frac{\xi}{i m}\right]_{2 \pi}^{4 \pi} \\
I^{2}= & {\left[-2 \cdot 1 \frac{\xi}{(i m)^{2}}+\frac{\xi^{2}}{i m}\right]_{2 \pi}^{4 \pi}, } \\
I^{3}= & {\left[3 \cdot 2 \cdot 1 \frac{\xi}{(i m)^{3}}-3 \cdot \frac{\xi^{2}}{(i m)^{2}}+\frac{\xi^{3}}{i m}\right]_{2 \pi}^{4 \pi}, } \\
I^{4}= & {\left[-4 \cdot 3 \cdot 2 \cdot 1 \frac{\xi}{(i m)^{4}}+4 \cdot 3 \frac{\xi^{2}}{(i m)^{3}}-4 \frac{\xi^{3}}{(i m)^{2}}+\frac{\xi^{4}}{i m}\right]_{2 \pi}^{4 \pi} } \\
& \cdots \ldots \ldots \cdots \cdots \cdot \ldots \cdot \ldots) \\
I^{\ell}= & {\left[\sum_{k=1}^{\ell}(-1)^{\ell-k} \frac{\ell ! \xi^{k}}{k !(i m)^{\ell-k+1}}\right]_{2 \pi}^{4 \pi} . }
\end{aligned}\right.
$$


It is also

$$
I^{\ell}=\left[\frac{1}{(i m)^{\ell+1}} \sum_{k=1}^{\ell}(-1)^{\ell-k} \frac{\ell !(\xi /(i m))^{k}}{k !}\right]_{2 \pi}^{4 \pi} .
$$

By explicit computations it follows that:

$$
\begin{aligned}
I^{\ell} & =\left[\sum_{k=1}^{\ell}(-1)^{\ell-k} \frac{\ell !(4 \pi)^{k}}{k !(i m)^{\ell-k+1}}\right]-\left[\sum_{k=1}^{\ell}(-1)^{\ell-k} \frac{\ell !(2 \pi)^{k}}{k !(i m)^{\ell-k+1}}\right] \\
& =\sum_{k=1}^{\ell}(-1)^{\ell-k} \frac{\ell !\left[(4 \pi)^{k}-(2 \pi)^{k}\right]}{k !(i m)^{\ell-k+1}}=\sum_{k=1}^{\ell}(-1)^{\ell-k} \frac{\ell !(2 \pi)^{k}\left(2^{k}-1\right)}{k !(i m)^{\ell-k+1}} .
\end{aligned}
$$

Analogously, it can be shown

Corollary 1. For a given $m \in \mathbb{Z} /\{0\}$ and $\ell \in \mathbb{N} \cup\{0\}$, it is

$$
\int_{-4 \pi}^{-2 \pi} \xi^{\ell} e^{-i m \xi} \mathrm{d} \xi=\sum_{k=1}^{\ell}(-1)^{k} \frac{\ell !(2 \pi)^{k}\left(2^{k}-1\right)}{k !(i m)^{\ell-k+1}}, \quad \ell \geq 1
$$

and

$$
\int_{-4 \pi}^{-2 \pi} \xi^{\ell} e^{-i m \xi} \mathrm{d} \xi=0, \quad \ell=0
$$

\section{Shannon Wavelets}

The real part (2.2) of the harmonic scaling functions (2.1), with half period, coincides with the sinc-function,

$$
\operatorname{sinc} x \stackrel{\text { def }}{=} \frac{\sin \pi x}{\pi x} .
$$

This function, is the mother function of the so-called Shannon multiresolution analysis. We must take as scaling function (Fig. 1.)

$$
\varphi(x) \stackrel{\text { def }}{=} \frac{\sin \pi x}{\pi x}=\frac{e^{\pi i x}-e^{-\pi i x}}{2 \pi i x},
$$

so that for the dilated and scaled instances, it is:

$$
\begin{aligned}
\varphi_{k}^{n}(x) & \stackrel{\text { def }}{=} 2^{n / 2} \varphi\left(2^{n} x-k\right)=2^{n / 2} \frac{\sin \pi\left(2^{n} x-k\right)}{\pi\left(2^{n} x-k\right)}= \\
& =2^{n / 2} \frac{e^{\pi i\left(2^{n} x-k\right)}-e^{-\pi i\left(2^{n} x-k\right)}}{2 \pi i\left(2^{n} x-k\right)} .
\end{aligned}
$$




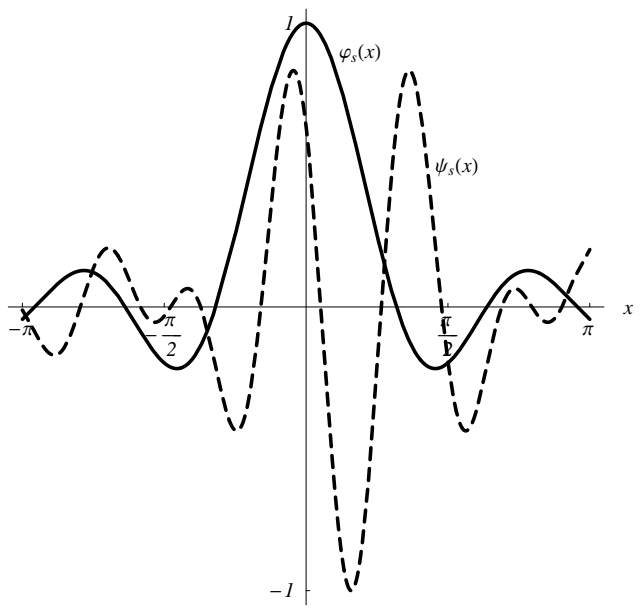

Figure 1. Shannon scaling function $\varphi(x)$ (thick line) and wavelet (dashed line) $\psi(x)$.

The Shannon wavelet is (see Fig. 1)

$$
\psi(x) \stackrel{\text { def }}{=} \frac{\sin \pi\left(x-\frac{1}{2}\right)-\sin 2 \pi\left(x-\frac{1}{2}\right)}{\pi\left(x-\frac{1}{2}\right)}
$$

i.e.

$$
\psi(x)=\frac{e^{-2 i \pi x}\left(-i+e^{i \pi x}+e^{3 i \pi x}+i e^{4 i \pi x}\right)}{(\pi-2 \pi x)} .
$$

It fulfills the condition

$$
\psi(x)=\varphi\left(x-\frac{1}{2}\right)-2 \varphi(2 x-1)
$$

and the dilated and scaled instances $\psi_{k}^{n}(x) \stackrel{\text { def }}{=} 2^{n / 2} \psi\left(2^{n} x-k\right)$ are

$$
\psi_{k}^{n}(x) \stackrel{\text { def }}{=} 2^{n / 2} \frac{\sin \pi\left(2^{n} x-k-\frac{1}{2}\right)-\sin 2 \pi\left(2^{n} x-k-\frac{1}{2}\right)}{\pi\left(2^{n} x-k-\frac{1}{2}\right)} .
$$

The Fourier transform of the scaling and wavelet function are respectively

$$
\widehat{\varphi}(\omega)=\frac{1}{2 \pi} \chi(\omega+3 \pi)
$$

i.e.

$$
\widehat{\varphi}(\omega)=\left\{\begin{array}{l}
1 /(2 \pi), \quad-\pi \leq \omega<\pi, \\
0, \quad \text { elsewhere }
\end{array}\right.
$$

and 


$$
\widehat{\psi}(\omega)=-e^{-i \omega / 2}[\chi(2 \omega)+\chi(-2 \omega)]
$$

being, explicitly,

$$
\widehat{\psi}(\omega)=\left\{\begin{array}{l}
-e^{-i \omega / 2}, \quad \pi \leq|\omega|<2 \pi \\
0, \quad \text { elsewhere }
\end{array}\right.
$$

It follows that the Fourier transform of the whole family of wavelets is given by

$$
\widehat{\psi}_{k}^{n}(\omega)=\frac{2^{-n / 2}}{2 \pi} e^{-i \omega k / 2^{n}} \widehat{\psi}\left(\omega / 2^{n}\right)
$$

i.e.

$$
\widehat{\psi}_{k}^{n}(\omega)=-\frac{2^{-n / 2}}{2 \pi} e^{-i \omega(k+1 / 2) / 2^{n}}\left[\chi\left(\omega / 2^{n-1}\right)+\chi\left(-\omega / 2^{n-1}\right)\right] .
$$

Analogously, according to (2.7) and (3.3) we have for the whole family of scaling functions:

$$
\widehat{\varphi}_{k}^{n}(\omega)=\frac{2^{-n / 2}}{2 \pi} e^{-i \omega k / 2^{n}} \chi\left(\omega / 2^{n}+3 \pi\right) .
$$

With the above expressions and the Parseval identity (2.8), we can easily show that

Theorem 3. Shannon wavelets are orthonormal functions, in the sense that

$$
\left\langle\psi_{k}^{n}(x), \psi_{h}^{m}(x)\right\rangle=\delta^{n m} \delta_{h k}
$$

$\delta^{n m}, \delta_{h k}$ being the Kronecker symbols.

Proof.

$$
\begin{aligned}
\left\langle\psi_{k}^{n}(x), \psi_{h}^{m}(x)\right\rangle & =2 \pi\left\langle\widehat{\psi}_{k}^{n}(\omega), \widehat{\psi}_{h}^{m}(\omega)\right\rangle \\
& =2 \pi \int_{-\infty}^{\infty} \frac{2^{-n / 2}}{2 \pi} e^{-i \omega(k+1 / 2) / 2^{n}}\left[\chi\left(\omega / 2^{n-1}\right)+\chi\left(-\omega / 2^{n-1}\right)\right] \\
& \times \frac{2^{-m / 2}}{2 \pi} e^{i \omega(h+1 / 2) / 2^{m}}\left[\chi\left(\omega / 2^{m-1}\right)+\chi\left(-\omega / 2^{m-1}\right)\right] \mathrm{d} \omega \\
& =\frac{2^{-(n+m) / 2}}{2 \pi} \int_{-\infty}^{\infty} e^{-i \omega(k+1 / 2) / 2^{n}+i \omega(h+1 / 2) / 2^{m}} \\
& \times\left[\chi\left(\omega / 2^{n-1}\right)+\chi\left(-\omega / 2^{n-1}\right)\right]\left[\chi\left(\omega / 2^{m-1}\right)+\chi\left(-\omega / 2^{m-1}\right)\right] \mathrm{d} \omega
\end{aligned}
$$

which is zero for $n \neq m$. For $n=m$ it is 


$$
\left\langle\psi_{k}^{n}(x), \psi_{h}^{n}(x)\right\rangle=\frac{2^{-n}}{2 \pi} \int_{-\infty}^{\infty} e^{-i \omega(h-k) / 2^{n}}\left[\chi\left(\omega / 2^{n-1}\right)+\chi\left(-\omega / 2^{n-1}\right)\right] \mathrm{d} \omega
$$

and, according to (2.4), by the change of variable $\xi=\omega / 2^{n-1}$

$$
\left\langle\psi_{k}^{n}(x), \psi_{h}^{n}(x)\right\rangle=\frac{1}{4 \pi}\left[\int_{-4 \pi}^{-2 \pi} e^{-2 i(h-k) \xi} \mathrm{d} \xi+\int_{2 \pi}^{4 \pi} e^{-2 i(h-k) \xi} \mathrm{d} \xi\right] .
$$

For $h=k($ and $n=m)$, is trivially

$$
\left\langle\psi_{k}^{n}(x), \psi_{k}^{n}(x)\right\rangle=1 .
$$

For $h \neq k$, it is

$$
\int_{2 \pi}^{4 \pi} e^{-2 i(h-k) \xi} \mathrm{d} \xi=\frac{i}{2(h-k)}\left(e^{-4 i \pi(h-k)}-e^{-8 i \pi(h-k)}\right)=0,
$$

and analogously $\int_{-4 \pi}^{-2 \pi} e^{-2 i(h-k) \xi} \mathrm{d} \xi=0$.

Moreover we have

Theorem 4. The translated instances of the Shannon scaling functions $\varphi_{k}^{n}(x)$, at the level $n=0$, are orthogonal, in the sense that

$$
\left\langle\varphi_{k}^{0}(x), \varphi_{h}^{0}(x)\right\rangle=\delta_{k h}
$$

being $\varphi_{k}^{0}(x) \stackrel{\text { def }}{=} \varphi(x-k)$.

Proof. It is

$$
\begin{aligned}
& \left\langle\varphi_{k}^{n}(x), \varphi_{h}^{m}(x)\right\rangle=2 \pi\left\langle\widehat{\varphi}_{k}^{n}(\omega), \widehat{\varphi}_{h}^{m}(\omega)\right\rangle= \\
& =2 \pi \int_{-\infty}^{\infty} \frac{2^{-n / 2}}{2 \pi} e^{-i \omega k / 2^{n}} \chi\left(\omega / 2^{n}+3 \pi\right) \frac{2^{-m / 2}}{2 \pi} e^{i \omega h / 2^{m}} \chi\left(\omega / 2^{m}+3 \pi\right) \mathrm{d} \omega \\
& =\frac{2^{-(n+m) / 2}}{2 \pi} \int_{-\infty}^{\infty} e^{-i \omega\left(k / 2^{n}-h / 2^{m}\right)} \chi\left(\omega / 2^{n}+3 \pi\right) \chi\left(\omega / 2^{m}+3 \pi\right) \mathrm{d} \omega .
\end{aligned}
$$

When $m=n$, we have

$$
\left\langle\varphi_{k}^{n}(x), \varphi_{h}^{n}(x)\right\rangle=\frac{2^{-n}}{2 \pi} \int_{-2^{n} \pi}^{2^{n} \pi} e^{-i \omega(k-h) / 2^{n}} \mathrm{~d} \omega=\frac{\sin [(h-k) \pi]}{(h-k) \pi} .
$$


Since $h, k \in \mathbb{Z}$, it follows that

$$
\frac{\sin [(h-k) \pi]}{(h-k) \pi}=\left\{\begin{array}{ll}
1, & h=k \\
0, & h \neq k
\end{array}\right\}=\delta_{k h}
$$

that is

$$
\left\langle\varphi_{k}^{n}(x), \varphi_{h}^{n}(x)\right\rangle=\delta_{k h} .
$$

When $m \neq n$, let say $m<n$, we have

$$
\left\langle\varphi_{k}^{n}(x), \varphi_{h}^{m}(x)\right\rangle=\frac{2^{-(n+m) / 2}}{2 \pi} \int_{-2^{m} \pi}^{2^{m} \pi} e^{-i \omega\left(k / 2^{n}-h / 2^{m}\right)} \mathrm{d} \omega,
$$

that is,

$$
\left\langle\varphi_{k}^{n}(x), \varphi_{h}^{m}(x)\right\rangle=2^{(m+n) / 2} \frac{\sin \left[\left(h-2^{m-n} k\right) \pi\right]}{\left(h-2^{m-n} k\right) \pi} .
$$

When $m \neq n$, the last expression is always different from zero, in fact (since $m<n)$

$$
\sin \left[\left(h-\frac{k}{2^{|m-n|}}\right) \pi\right]=0 \Rightarrow\left[h-\frac{k}{2^{|m-n|}}\right] \pi=s \pi, \quad s \in \mathbb{Z},
$$

i.e.

$$
h=s+\frac{k}{2^{|m-n|}}, \quad h, k, s \in \mathbb{Z}
$$

and $h \in \mathbb{Z}$ only if $m=n$. Therefore, in order to have the orthogonality the equality $m=n$ should be satisfied. So that

$$
\left\langle\varphi_{k}^{n}(x), \varphi_{h}^{n}(x)\right\rangle=2^{n} \delta_{k h},
$$

and, in particular, when $n=0$,

$$
\left\langle\varphi_{k}^{0}(x), \varphi_{h}^{0}(x)\right\rangle=\delta_{k h} .
$$

The scalar product of the Shannon scaling functions with respect to the corresponding wavelets is characterized by the following

Theorem 5. The translated instances of the Shannon scaling functions $\varphi_{k}^{n}(x)$, at the level $n=0$, are orthogonal to the Shannon wavelets, in the sense that

$$
\left\langle\varphi_{k}^{0}(x), \psi_{h}^{m}(x)\right\rangle=0, \quad m \geq 0,
$$

being $\varphi_{k}^{0}(x) \stackrel{\text { def }}{=} \varphi(x-k)$. 
Proof. It is

$$
\begin{aligned}
& \left\langle\varphi_{k}^{n}(x), \psi_{h}^{m}(x)\right\rangle=2 \pi\left\langle\widehat{\varphi}_{k}^{n}(\omega), \widehat{\psi}_{h}^{m}(\omega)\right\rangle \\
& =2 \pi \int_{-\infty}^{\infty} 2^{-n / 2} e^{-i \omega k / 2^{n}} \chi\left(\omega / 2^{n}+3 \pi\right) \frac{2^{-m / 2}}{2 \pi} e^{i \omega(h+1 / 2) / 2^{m}} \\
& \quad \times\left[\chi\left(\omega / 2^{m-1}\right)+\chi\left(-\omega / 2^{m-1}\right)\right] \mathrm{d} \omega \\
& =2^{-(n+m) / 2} \int_{-\infty}^{\infty} e^{-i \omega k / 2^{n}+i \omega(h+1 / 2) / 2^{m}} \chi\left(\omega / 2^{n}+3 \pi\right) \\
& \quad \times\left[\chi\left(\omega / 2^{m-1}\right)+\chi\left(-\omega / 2^{m-1}\right)\right] \mathrm{d} \omega,
\end{aligned}
$$

which is zero for $m \geq n \geq 0$ (since, according to (2.4), the compact support of the characteristic functions do not intersect). On the contrary, it can be easily seen that, for $m<n$ it is

$$
\begin{aligned}
\left\langle\varphi_{k}^{n}(x), \psi_{h}^{m}(x)\right\rangle & =2^{-(n+m) / 2} \int_{2^{m} \pi}^{2^{n} \pi} e^{-i \omega k / 2^{n}+i \omega(h+1 / 2) / 2^{m}} \mathrm{~d} \omega \\
& =-\frac{2^{1+(m+n) / 2}\left(i e^{i \pi\left[2^{-m+n-1}(1+2 h)-k\right]}+e^{i \pi\left(h-2^{m-n} k\right)}\right)}{2^{n}(1+2 h)-2^{1+m} k}
\end{aligned}
$$

and this product, in general does not vanish.

\section{Connection Coefficients for the Shannon Wavelets}

Any order connection coefficients of the wavelets, are defined as

$$
\gamma^{(\ell)} \underset{k h m}{\stackrel{\text { def }}{=}}\left\langle\frac{\mathrm{d}^{\ell}}{\mathrm{d} x^{\ell}} \psi_{k}^{n}(x), \psi_{h}^{m}(x)\right\rangle .
$$

They can be easily computed by the following theorem (for the first and second order connection coefficients of periodic harmonic wavelets see also [2, 4, 13]). In the Fourier domain the $\ell$-order derivative of the wavelet basis is

$$
\frac{\mathrm{d}^{\ell}}{\mathrm{d} x^{\ell}} \psi_{k}^{n}(x)=(i \omega)^{\ell} \widehat{\psi}_{k}^{n}(\omega)
$$

and according to (3.6)

$$
\begin{aligned}
\frac{\mathrm{d}^{\ell}}{\mathrm{d} x^{\ell}} \psi_{k}^{n}(x) & =(i \omega)^{\ell} \frac{2^{-n / 2}}{2 \pi} e^{-i \omega k / 2^{n}} \widehat{\psi}\left(\omega / 2^{n}\right) \\
& =-(i \omega)^{\ell} \frac{2^{-n / 2}}{2 \pi} e^{-i \omega(k+1 / 2) / 2^{n}}\left[\chi\left(\omega / 2^{n-1}\right)+\chi\left(-\omega / 2^{n-1}\right)\right] .
\end{aligned}
$$


Any order connection coefficients, taking into account the definition (4.1), are equivalently

$$
\gamma_{k h}^{(\ell) \underset{n m}{\text { def }}=}\left\langle\frac{\mathrm{d}^{\ell}}{\mathrm{d} x^{\ell}} \psi_{k}^{n}(x), \psi_{h}^{m}(x)\right\rangle=2 \pi\left\langle\frac{\mathrm{d}^{\ell}}{\mathrm{d} x^{\ell}} \psi_{k}^{n}(x), \widehat{\psi_{h}^{m}(x)}\right\rangle .
$$

Starting from equations (4.2), (4.3), the connection coefficient (4.1) can be computed according to

Theorem 6. The connection coefficients (4.1) of the Shannon wavelets (3.5) are given by

$$
\begin{aligned}
& \gamma_{k h}^{(\ell) n m}=\frac{i^{\ell} 2^{n \ell}}{2 \pi}\left\{\delta_{k h}\left[1+(-1)^{\ell}\right] \frac{\pi^{\ell+1}}{\ell+1}\left(2^{\ell+1}-1\right)\right. \\
& \left.+\left(1-\delta_{k h}\right)^{\ell+1} \sum_{j=1}^{\ell}(-1)^{\ell} \frac{\ell ! \pi^{j}\left(2^{j}-1\right)}{j ![i(h-k)]^{\ell-j+1}}\left[(-1)^{j}-1\right]\right\} \delta^{n m}
\end{aligned}
$$

for $\ell \geq 1$, and $\gamma_{k h}^{(\ell) n m}=\delta_{k h} \delta^{n m}$, when $\ell=0$.

Proof. From their definition (4.1), taking into account equations (2.8)-(4.3), we get

$$
\begin{aligned}
\left\langle\frac{\mathrm{d}^{\ell}}{\mathrm{d} x^{\ell}} \psi_{k}^{n}(x), \psi_{h}^{m}(x)\right\rangle & =2 \pi\left\langle\frac{\mathrm{d}^{\ell}}{\mathrm{d} x^{\ell}} \psi_{k}^{n}(x), \widehat{\psi_{h}^{m}(x)}\right\rangle=2 \pi\left\langle(i \omega)^{\ell} \widehat{\psi}_{k}^{n}(\omega), \widehat{\psi}_{h}^{m}(\omega)\right\rangle \\
& =2 \pi \int_{-\infty}^{\infty} \frac{2^{-n / 2}}{2 \pi}(i \omega)^{\ell} e^{-i \omega(k+1 / 2) / 2^{n}}\left[\chi\left(\omega / 2^{n-1}\right)+\chi\left(-\omega / 2^{n-1}\right)\right] \\
& \times \frac{2^{-m / 2}}{2 \pi} e^{i \omega(h+1 / 2) / 2^{m}}\left[\chi\left(\omega / 2^{m-1}\right)+\chi\left(-\omega / 2^{m-1}\right)\right] \mathrm{d} \omega
\end{aligned}
$$

which is zero when $m \neq n$. When $m=n$, it follows that

$$
\gamma_{k h}^{(\ell) n n}=\frac{2^{-n}}{2 \pi} \int_{-\infty}^{\infty}(i \omega)^{\ell} e^{-i \omega(k-h) / 2^{n}}\left[\chi\left(\omega / 2^{n-1}\right)+\chi\left(-\omega / 2^{n-1}\right)\right] \mathrm{d} \omega
$$

and, with the change of variable $\omega / 2^{n-1}=\xi$,

$$
\gamma_{k h}^{(\ell) n n}=\frac{i^{\ell} 2^{(n-1) \ell}}{4 \pi} \int_{-\infty}^{\infty} \xi^{\ell} e^{-i \xi(k-h) / 2}[\chi(\xi)+\chi(-\xi)] \mathrm{d} \xi
$$

i.e.

$$
\gamma_{k h}^{(\ell)} \underset{k n}{n n}=\frac{i^{\ell} 2^{(n-1) \ell}}{4 \pi}\left[\int_{-\infty}^{\infty} \xi^{\ell} e^{-i \xi(k-h) / 2} \chi(\xi) \mathrm{d} \xi+\int_{-\infty}^{\infty} \xi^{\ell} e^{-i \xi(k-h) / 2} \chi(-\xi) \mathrm{d} \xi\right] .
$$


From where, if we notice that

$$
\int_{-\infty}^{\infty} \ldots \chi(\xi) \mathrm{d} \xi=\int_{2 \pi}^{4 \pi} \ldots \mathrm{d} \xi, \quad \int_{-\infty}^{\infty} \ldots \chi(-\xi) \mathrm{d} \xi=\int_{-4 \pi}^{-2 \pi} \ldots \mathrm{d} \xi
$$

by taking into account equations (2.9)-(2.12) there easily follows the value of the connection coefficients. Alternatively from (4.7) with the change $-\xi \rightarrow \xi$ in the second integral

$$
\gamma_{k h}^{(\ell) n n}=\frac{i^{\ell} 2^{(n-1) \ell}}{4 \pi}\left[\int_{-\infty}^{\infty} \xi^{\ell} e^{-i \xi(k-h) / 2} \chi(\xi) \mathrm{d} \xi-\int_{-\infty}^{\infty}(-\xi)^{\ell} e^{i \xi(k-h) / 2} \chi(\xi) \mathrm{d} \xi\right],
$$

we obtain

$$
\gamma_{k h}^{(\ell) n n}=\frac{i^{\ell} 2^{(n-1) \ell}}{4 \pi} \int_{2 \pi}^{4 \pi} \xi^{\ell}\left[e^{-i \xi(k-h) / 2}+(-1)^{\ell} e^{i \xi(k-h) / 2}\right] \mathrm{d} \xi .
$$

Although the imaginary unit is present as a factor, the connection coefficients are real values. In fact, by transforming the integral into trigonometric functions we get

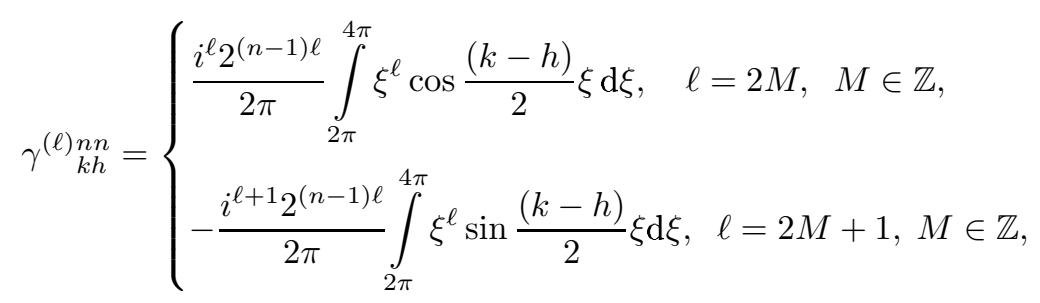

being $i^{\ell}= \pm 1$ for $\ell=2 M, M \in \mathbb{Z}$, and $i^{\ell}= \pm i$ for $\ell=2 M+1, M \in \mathbb{Z}$. When $k=h$, we obtain from $(4.8)$

$\gamma_{k k}^{(\ell) n n}=\frac{i^{\ell} 2^{(n-1) \ell}}{2 \pi(\ell+1)}\left(1+(-1)^{\ell}\right)\left[\xi^{\ell+1}\right]_{2 \pi}^{4 \pi}=\frac{i^{\ell} 2^{n \ell} \pi^{\ell+1}}{2 \pi(\ell+1)}\left[\left(1+(-1)^{\ell}\right)\left(2^{\ell+1}-1\right)\right]$.

When $k \neq h$, from (4.8) by taking into account equations (2.9)-(2.12) we have

$$
\gamma_{k h}^{(\ell) n n}=\frac{i^{\ell} 2^{(n-1) \ell}}{4 \pi} \sum_{j=1}^{\ell}(-1)^{\ell} \frac{\ell !(2 \pi)^{j}\left(2^{j}-1\right) 2^{\ell-j+1}}{j ![i(h-k)]^{\ell-j+1}}\left[(-1)^{j}-1\right], \quad \ell \geq 1,
$$

i.e.

$$
\gamma_{k h}^{(\ell)}=\frac{i^{\ell} 2^{n \ell}}{2 \pi} \sum_{j=1}^{\ell}(-1)^{\ell} \frac{\ell ! \pi^{j}\left(2^{j}-1\right)}{j ![i(h-k)]^{\ell-j+1}}\left[(-1)^{j}-1\right], \quad \ell \geq 1 .
$$

When $\ell$ is even and $j$ is an odd number, the denominator gives a real number, so that its ratio with $i^{\ell}$ is a real number. When $\ell$ is odd and $j$ is an even number 
the connection coefficients are null. When $\ell$ and $j$ are both odd numbers, the denominator gives a complex number so that the ratio with $i^{\ell}$ is a real number. Thus the connection coefficients of Shannon wavelets are always real numbers.

The second part of the proof is easily obtained by using Theorem 3, i.e. by noticing that when $\ell=0$ the connection coefficients reduce to the scalar product of the Shannon wavelet.

In particular, by assuming $k=0, \ldots, 2^{n}-1, h=0, \ldots, 2^{m}-1$, from (4.5), we have explicitly:

- For the first order $(\ell=1)$ coefficients $\gamma_{k h}^{(1) n m}$, at the lower scales $0 \leq n=$ $m \leq 3$ are the following

$$
\begin{aligned}
& \gamma_{00}^{(1) 00}=0, \quad \gamma_{k h}^{(1)}{ }_{k h}^{11}=4\left(\begin{array}{cc}
0 & \frac{1}{2} \\
-\frac{1}{2} & 0
\end{array}\right), \quad \gamma_{k h}^{(1) 22}=4\left(\begin{array}{cccc}
0 & 1 & \frac{1}{2} & \frac{1}{3} \\
-1 & 0 & 1 & \frac{1}{2} \\
-\frac{1}{2} & -1 & 0 & 1 \\
-\frac{1}{3} & -\frac{1}{2} & -1 & 0
\end{array}\right), \\
& \gamma_{k h}^{(1) 33}=4\left(\begin{array}{cccccccc}
0 & 2 & 1 & \frac{2}{3} & \frac{1}{2} & \frac{2}{5} & \frac{1}{3} & \frac{2}{7} \\
-2 & 0 & 2 & 1 & \frac{2}{3} & \frac{1}{2} & \frac{2}{5} & \frac{1}{3} \\
-1 & -2 & 0 & 2 & 1 & \frac{2}{3} & \frac{1}{2} & \frac{2}{5} \\
-\frac{2}{3} & -1 & -2 & 0 & 2 & 1 & \frac{2}{3} & \frac{1}{2} \\
-\frac{1}{2} & -\frac{2}{3} & -1 & -2 & 0 & 2 & 1 & \frac{2}{3} \\
-\frac{2}{5} & -\frac{1}{2} & -\frac{2}{3} & -1 & -2 & 0 & 2 & 1 \\
-\frac{1}{3} & -\frac{2}{5} & -\frac{1}{2} & -\frac{2}{3} & -1 & -2 & 0 & 2 \\
-\frac{2}{7} & -\frac{1}{3} & -\frac{2}{5} & -\frac{1}{2} & -\frac{2}{3} & -1 & -2 & 0
\end{array}\right) .
\end{aligned}
$$

- For the second order $(\ell=2)$ coefficients $\gamma_{k h}^{(1) n m}$, at the lower scales $0 \leq$ $n=m \leq 3$ :

$$
\begin{aligned}
& \gamma_{00}^{(2) 00}=\frac{-7 \pi^{2}}{3}, \quad \gamma_{k h}^{(2) 11}=\left(\begin{array}{cc}
\frac{-28 \pi^{2}}{3} & -8 \\
-8 & \frac{-28 \pi^{2}}{3}
\end{array}\right), \\
& \gamma_{k h}^{(2) 22}=\left(\begin{array}{cccc}
\frac{-112 \pi^{2}}{3} & -32 & -8 & -\frac{32}{9} \\
-32 & \frac{-112 \pi^{2}}{3} & -32 & -8 \\
-8 & -32 & \frac{-112 \pi^{2}}{3} & -32 \\
-\frac{32}{9} & -8 & -32 & \frac{-112 \pi^{2}}{3}
\end{array}\right) \text {, } \\
& \gamma_{k h}^{(2) 33}=\left(\begin{array}{cccccccc}
\frac{-448 \pi^{2}}{3} & -128 & -32 & -\frac{128}{9} & -8 & -\frac{128}{25} & -\frac{32}{9} & -\frac{128}{49} \\
-128 & \frac{-448 \pi^{2}}{3} & -128 & -32 & -\frac{128}{9} & -8 & -\frac{128}{25} & -\frac{32}{9} \\
-32 & -128 & \frac{-448 \pi^{2}}{3} & -128 & -32 & -\frac{128}{9} & -8 & -\frac{128}{25} \\
-\frac{128}{9} & -32 & -128 & \frac{-448 \pi^{2}}{3} & -128 & -32 & -\frac{128}{9} & -8 \\
-8 & -\frac{128}{9} & -32 & -128 & \frac{-448 \pi^{2}}{3} & -128 & -32 & -\frac{128}{9} \\
-\frac{128}{25} & -8 & -\frac{128}{9} & -32 & -128 & \frac{-448 \pi^{2}}{3} & -128 & -32 \\
-\frac{32}{9} & -\frac{128}{25} & -8 & -\frac{128}{9} & -32 & -128 & \frac{-448 \pi^{2}}{3} & -128 \\
-\frac{128}{49} & -\frac{32}{9} & -\frac{128}{25} & -8 & -\frac{128}{9} & -32 & -128 & \frac{-448 \pi^{2}}{3}
\end{array}\right) .
\end{aligned}
$$


- For the third order $(\ell=3)$ coefficients $\gamma_{k h}^{(1) n m}$, at the lower scales $0 \leq n=$ $m \leq 2$ :

$$
\begin{aligned}
& \gamma_{k h}^{(3)}=0, \quad \gamma_{k h}^{(3) 11}=\left(\begin{array}{cc}
0 & 48-56 \pi^{2} \\
-48+56 \pi^{2} & 0
\end{array}\right), \\
& \gamma_{k h}^{(3) 22}=\left(\begin{array}{cccc}
0 & -64\left(-6+7 \pi^{2}\right) & 48-224 \pi^{2} & \frac{-64\left(-2+21 \pi^{2}\right)}{9} \\
64\left(-6+7 \pi^{2}\right) & 0 & -64\left(-6+7 \pi^{2}\right) & 48-224 \pi^{2} \\
-48+224 \pi^{2} & 64\left(-6+7 \pi^{2}\right) & 0 & -64\left(-6+7 \pi^{2}\right) \\
\frac{64\left(-2+21 \pi^{2}\right)}{9} & -48+224 \pi^{2} & 64\left(-6+7 \pi^{2}\right) & 0
\end{array}\right)
\end{aligned}
$$

We have the following

Corollary 2. The connection coefficients of the Shannon wavelets have these symmetries with respect to the lower indices,

$$
\left\{\begin{array}{l}
\gamma_{k h}^{(\ell) n n}=\gamma_{h k}^{(\ell) n n}, \quad \ell=2 M \in \mathbb{Z}, \\
\gamma_{k h}^{(\ell) n n}=-\gamma_{h k}^{(\ell) n n}, \quad \ell=2 M+1 \in \mathbb{Z} .
\end{array}\right.
$$

Proof. It immediately follows from (4.5) by a simple computation.

\section{References}

[1] N.M. Astafeva. Wavelet analysis: basic theory and examples of applications. Usp. Phys. Nauk, 166(11), 1145-1170, 1996.

[2] C. Cattani. Harmonic wavelet solutions of the Schrödinger equation. International Journal of Fluid Mechanics Research, 5, 1-10, 2003.

[3] C. Cattani. Multiscale analysis of wave propagation in composite materials. Mathematical Modelling and Analysis, 8(4), 267-282, 2003.

[4] C. Cattani. The wavelets based technique in the dispersive wave propagation. International Applied Mechanics, 39(4), 132-140, 2003.

[5] C. Cattani and S.V. Rogosin. Harmonic wavelets and their certain properties. In: Proceedings of the Belarussian Academy of Science, ser. PhysicalMathematical Sciences, Izv. Nat. Akad. Nauk Belarusi, volume 2, 35-41, 2005.

[6] C.Cattani. Harmonic wavelets towards solution of nonlinear PDE. Computers and Mathematics with Applications, 50(8-9), 1191-1210, 2005.

[7] W. Dahmen. Wavelet and multiscale methods for operator equations. Acta Numerica, 6, 55-228, 1997.

[8] W. Dahmen and C.A. Micchelli. Using the refinement equation for evaluating integrals of wavelets. Siam J. Numer. Anal., 30, 507-537, 1993.

[9] I. Daubechies. Ten Lectures on wavelets. SIAM, Philadelphia, 1992.

[10] A. Latto, H.L. Resnikoff and E. Tenenbaum. The evaluation of connection coefficients of compactly supported wavelets. In: Y. Maday(Ed.), Proc. of the French-USA workshop on wavelets and turbulence. Springer-Verlag, 76-89, 1992.

[11] E.B. Lin and X. Zhou. Connection coefficients on an interval and wavelet solutions of Burgers equation. Journal of Computational and Applied Mathematics, 135, 63-78, 2001. 
[12] H. Mouri and H. Kubotani. Real-valued harmonic wavelets. Phys.Lett. A, 201, 53-60, 1995.

[13] S.V. Muniandy and I.M. Moroz. Galerkin modelling of the Burgers equation using harmonic wavelets. Phys.Lett. A, 235, 352-356, 1997.

[14] D.E. Newland. Harmonic wavelet analysis. In: Proc.R.Soc.Lond. A, volume 443, 203-222, 1993. 